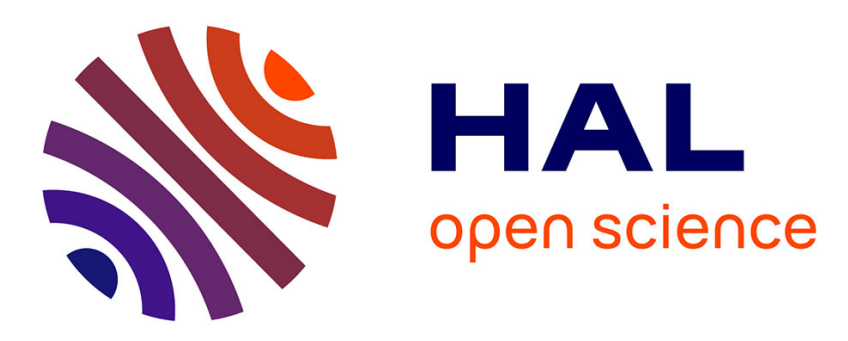

\title{
A-Type Crystals from Dilute Solutions of Short Amylose Chains
}

Nicole Montesanti, Gabrielle Veronese, Alain Buleon, Pierre Escalier, Shinichi Kitamura, Jean-Luc Putaux

\section{- To cite this version:}

Nicole Montesanti, Gabrielle Veronese, Alain Buleon, Pierre Escalier, Shinichi Kitamura, et al.. AType Crystals from Dilute Solutions of Short Amylose Chains. Biomacromolecules, 2010, 11 (11), pp.3049-3058. 10.1021/bm1008712 . hal-02666838

\section{HAL Id: hal-02666838 \\ https://hal.inrae.fr/hal-02666838}

Submitted on 30 Nov 2021

HAL is a multi-disciplinary open access archive for the deposit and dissemination of scientific research documents, whether they are published or not. The documents may come from teaching and research institutions in France or abroad, or from public or private research centers.
L'archive ouverte pluridisciplinaire HAL, est destinée au dépôt et à la diffusion de documents scientifiques de niveau recherche, publiés ou non, émanant des établissements d'enseignement et de recherche français ou étrangers, des laboratoires publics ou privés. 


\title{
A-type crystals from dilute solutions of short amylose chains
}

\author{
Nicole Montesanti ${ }^{1}$, Gabrielle Véronèse ${ }^{2,3}$, Alain Buléon ${ }^{4}$, Pierre-Claude Escalier ${ }^{2,3}$, \\ Shinichi Kitamura ${ }^{5}$ and Jean-Luc Putaux ${ }^{1 *}$
}

${ }^{1}$ Centre de Recherches sur les Macromolécules Végétales (CERMAV-CNRS), BP 53, F-38041 Grenoble Cedex 9, France - affiliated with Université Joseph Fourier and member of the Institut de Chimie Moléculaire de Grenoble

${ }^{2}$ Université de Toulouse, INSA-UPS-INP, LISBP, 135 Avenue de Rangueil, F-31077 Toulouse, France

${ }^{3}$ UMR 5504, UMR 792 Ingénierie des Systèmes Biologiques et des Procédés, CNRS, INRA, F-31400 Toulouse, France

${ }^{4}$ Unité Biopolymères Interactions Assemblages, Institut National de la Recherche Agronomique, Rue de la Géraudière, BP 71627, F-44316 Nantes Cedex 3, France.

${ }^{5}$ Graduate School of Life and Environmental Sciences, Osaka Prefecture University, Sakai, Osaka 599-8531, Japan

*corresponding author: jean-luc.putaux@cermav.cnrs.fr

Published in: Biomacromolecules 11 (2010), 3049-3058

DOI: 10.1021/bm1008712 


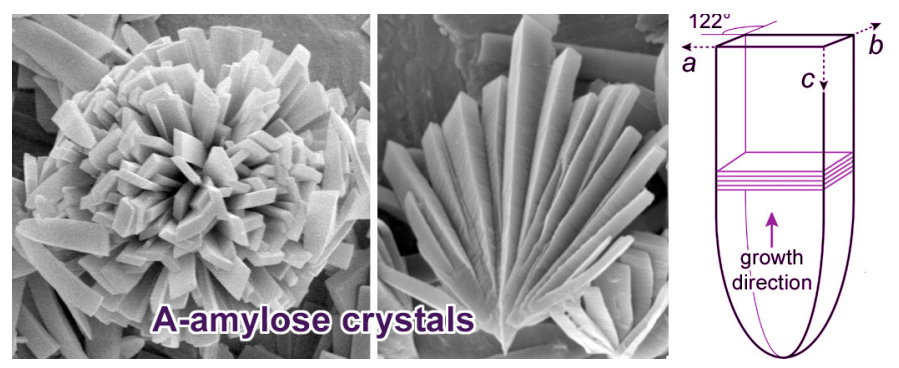

\begin{abstract}
Model A-type amylose single crystals were prepared by recrystallizing dextrins from acid-hydrolyzed native starch and narrow fractions of short chains of enzymaticallysynthesized amylose, in dilute water/acetone solutions. In most cases, spindle-shaped crystals with a sharp, round or flat apical end were formed, organized in rosettes or fan-like assemblies. The morphology and crystal size were shown to strongly depend on the average degree of polymerization $(\overline{D P})$, distribution width $(D W)$ and degree of branching of the chains. The largest and most clearly faceted single crystals were prepared using fractions of synthetic amylose. Typically, 5-10 $\mu \mathrm{m}$-long crystals were obtained from fractions with $17 \leq \overline{D P} \leq 20$ and $D W \leq 8$. Chains with $\overline{D P}>40$ and a high polydispersity formed ill-defined networks of smaller crystallites. Fractions of branched and more polydisperse limit dextrins yielded crystals smaller than those obtained from narrow fractions of synthetic amylose. The morphological analysis of faceted single crystals combined with electron diffraction data confirmed that the double helices were oriented along the long dimension of the crystal and packed into lamellae with a parallelogram cross section defined by the $a$ and $b$ directions of the monoclinic unit cell of A-amylose. The lamellae are stacked along the $c$-axis that is oriented parallel but opposite to the growth direction of the crystal.
\end{abstract}

Keywords: A-type amylose, single crystals, limit dextrins, synthetic amylose, amylosucrase, $\alpha$-glucan phosphorylase 


\section{Introduction}

Starch, the major energy reserve of a large variety of higher plants, is mainly composed of two polysaccharides, namely amylose and amylopectin. Amylose is described as a mostly linear polymer of $\alpha(1,4)$-connected D-glucopyranosyl units with less than $1 \% \alpha(1,6)$ branching linkages. Amylopectin is a highly branched macromolecule composed of $\alpha(1,4)$-D-glucan segments linked by $5-6 \% \alpha(1,6)$ bonds. ${ }^{1}$ Native starch granules exhibit two main allomorphic types which can be identified by X-ray scattering or solid state ${ }^{13} \mathrm{C} N M R .{ }^{1-3}$ The A-type mainly occurs in cereal starches and the B-type in tubers and amylose-rich starches. C-type granules, found for instance in legume starch, contain a mixture of A- and B-type crystallites.

Several authors have prepared model substrates in vitro and analyzed their diffraction data and thermal properties to understand the structural transitions occurring during starch processing and propose 3-D models of starch crystallites. Amylose can be recrystallized in vitro from solution into the $A$ and $B$ crystalline forms ${ }^{4}$ by cooling ${ }^{5,6}$ or adding a precipitant. ${ }^{7,8}$ The resulting morphology (gels, precipitates, spherulites, crystals, etc.) and allomorphic type ( $A$ or $B$ ) depend on factors such as solvent, molecular weight, branching, concentration and temperature. A general rule is that low crystallization temperatures and long chains induce the B form, whereas high concentrations, high temperatures and short chains favor A-type crystallization. ${ }^{4}$ Molecular models were built to describe the structure of the crystalline domains in starch granules using diffraction data collected from residues of acid-hydrolyzed granules, ${ }^{9}$ recrystallized fibers, ${ }^{10-12}$ or single crystals. ${ }^{13,14}$ In the most recent models of the $A$ and $B$ allomorphs, amylose exhibits a 6-fold left-handed double helical conformation with pitches of 2.08 and $2.13 \mathrm{~nm}$, respectively. ${ }^{10-14}$

The crystallization of amylose into lamellar crystals has been investigated using fractions with a well-defined degree of polymerization $(D P)$, obtained by using various procedures. One of them consists in performing a mild acid hydrolysis of native starch in order to isolate the crystalline fraction of the granules. ${ }^{7,13}$ The acid-resistant fraction is then solubilized and the resulting short chains are recrystallized in the presence of various precipitants. For instance, the acid hydrolysis of potato starch for $48 \mathrm{~h}$ at $65^{\circ} \mathrm{C}$ releases chains with an average $D P(\overline{D P})$ of 21 and only one $\alpha(1,6)$ branch. ${ }^{14}$ Short amylose chains can also be obtained by enzymatic debranching of glycogen, which yields $\overline{D P} 11$ linear chains, ${ }^{15}$ or synthesized in vitro by enzymes like phosphorylase ${ }^{16}$ and amylosucrase. ${ }^{17}$

Pfannemüller reported that, in aqueous solutions of amylose synthesized in vitro by phosphorylase and at room temperature, chains with $\overline{D P} 10-12$ and $\overline{D P} \geq 13$ crystallized into the A- and B-type, respectively. ${ }^{18}$ Single crystals were also prepared from dilute water/ethanol mixtures by precipitation of short chains stemming from acid hydrolysis or enzymatic synthesis. ${ }^{7}$ The nature of the resulting allomorph was shown to depend on ethanol 
concentration. B- and A-type crystals were obtained in pure water and 15\% ( $/ \mathrm{v})$ ethanol, respectively. ${ }^{7}$ Micron-sized oblong A-amylose crystals were produced from dilute $(0.05 \% \mathrm{w} / \mathrm{v})$ solutions of acid-hydrolyzed potato starch using a slow diffusion of acetone vapors at $60^{\circ} \mathrm{C}$ to induce precipitation. ${ }^{13}$ Electron diffraction diagrams recorded from individual crystals showed that the chain axis was parallel to the long axis of the crystal. The patterns were analyzed to propose a space group and refine the unit cell. ${ }^{13}$ Pohu et al. have shown that similar oblong crystals were made of stacks of parallel lamellae having an approximate thickness of 5-7 nm. ${ }^{19}$ More recently, an amylose fraction with $\overline{D P} 17$, prepared by in vitro synthesis by amylosucrase, was crystallized using the same protocol. The resulting 5-15 $\mu$ m-long A-amylose crystals were probed by a microbeam of synchrotron radiation. Single crystal X-ray datasets, collected up to a resolution of $0.13 \mathrm{~nm}$, were used to refine the unit cell ${ }^{20}$ and revisit the structure previously proposed by Imberty et al. ${ }^{21}$

In the present paper, we report on the in vitro crystallization of a series of fractions of shortchain amylose (limit dextrins from native starch and enzymatically-synthesized linear chains). The influence of their $D P$, polydispersity, and degree of branching on the morphology of the resulting crystals has been studied.

\section{Experimental Section}

Sources of Short Amylose-like Chains. Limit dextrins were prepared by mild acid hydrolysis of potato and wrinkled pea starch granules in $2.2 \mathrm{~N} \mathrm{HCl}$ at $35^{\circ} \mathrm{C}$ for 40 days, according to a method described elsewhere. ${ }^{22}$ They were used without further fractionation. The wrinkled pea and potato dextrin fractions were analyzed by high-performance anion-exchange chromatography with pulsed amperometric detection (HPAEC-PAD) (Figure 1a and Supporting Information, Figure S1, respectively). The DP ranged from 6 to 42 for wrinkled pea starch and from 8 to 27 for potato starch. In the following, the fractions of limit dextrins from wrinkled pea and potato starch will be referred to as LDWP6-42 and LDP8-27, respectively. They contained 4 and $5 \%$ residual $\alpha(1,6)$ branching points, respectively, as determined by ${ }^{1} \mathrm{H}$ NMR spectroscopy.

A third fraction of dextrins, kindly given by $A$. Heyraud (CERMAV), was prepared by preparative gel filtration of commercial maize maltodextrins (Glucidex $17^{\mathrm{TM}}$ from Roquette, Lestrem, France). The DP distribution of this fraction (ranging from 6 to 22) was determined by matrix-assisted laser desorption ionization - time of flight (MALDI-TOF) mass spectrometry (Figure S2a). The dextrins contained $8 \%$ residual $\alpha(1,6)$ branching points. In the following, the fraction of maltodextrins from maize will be referred to as MDM6-22.

Linear $\alpha(1,4)$-D-glucan chains were synthesized in vitro by amylosucrase. The purified glutathione-S-transferase / amylosucrase fusion protein was produced and purified as 
previously described. ${ }^{17}$ The synthesis reaction was performed at $30^{\circ} \mathrm{C}$ during $24 \mathrm{~h}$ in a $50 \mathrm{mM}$ Tris- $\mathrm{HCl}$ buffer $\mathrm{pH} 7.0$, from $600 \mathrm{mM}$ sucrose as glucosyl donor and $100 \mathrm{mM}$ maltose as acceptor, using $0.5 \mathrm{U} / \mathrm{mL}$ glutathione-S-transferase/amylosucrase. The total fraction, containing fructose and maltooligosaccharides of $D P$ ranging from 2 to 36 , was subsequently fractionated by preparative gel filtration. HPAEC-PAD was used to determine the DP distribution of each resulting narrow fraction. Some examples of chromatograms are given in Figure 1b. These fractions will be referred to as SAA (for "synthetic amylose / amylosucrase") with the indication of the minimum and maximum $D P s$ of the considered fraction. Fractions SAA12-23, SAA19-29 and SAA23-32 were obtained by fractionation by gel filtration using a $480 \mathrm{~mL}$ column of Biogel P6 Extra Fine (Biorad). Sugars were eluted at $60{ }^{\circ} \mathrm{C}$ in water using a flow-rate of $0.66 \mathrm{~mL} / \mathrm{min}$. Fractions SAA9-15, SAA14-21 and SAA16-22 were prepared using a $120 \mathrm{~mL}$ column containing Biogel P6 Fine (Biorad). Sugars were eluted at $60{ }^{\circ} \mathrm{C}$ in water using a flow-rate of $0.5 \mathrm{~mL} / \mathrm{min}$.

Two other fractions of longer linear amylose chains were synthesized in vitro using starch phosphorylase, according to the method described by Kitamura et al. ${ }^{16}$ Their DP distribution was determined by MALDI-TOF mass spectrometry (Figure S2b). In the following, these fractions will be referred to as SAP (for "synthetic amylose / phosphorylase"). We have used fractions SAP20-40 and SAP40-80. The nomenclature and characteristics of the fractions used in this study are summarized in Table 1.

High-Performance Anion-Exchange Chromatography (HPAEC). The characterization of the amylose chains contained in the SAA, LDWP6-42 and LDP8-27 fractions was performed by HPAEC-PAD on a $4 \times 250 \mathrm{~mm}$ Dionex Carbo-Pac PA100. The detection was performed using a Dionex ED40 module with a gold working electrode and a $\mathrm{Ag} / \mathrm{AgCl} \mathrm{pH}$ reference. The flow rate of $\mathrm{NaOH} 150 \mathrm{mM}$ was $1.0 \mathrm{~mL} / \mathrm{min}$ and an acetate gradient was applied as follows: 0-2 min: $115 \mathrm{mM}$; 2-12 min: 115-225 mM; 12-47 min: 225-300 mM; 47-48 min: 300-300 mM; 48-58 min: $115 \mathrm{mM}$. For each $D P_{i}$, the concentration of maltooligosaccharides $C_{i}$ was evaluated using the linear relationship between the detector response per mole of $\alpha(1,4)$ chains and its degree of polymerization. ${ }^{23}$ The linear curve coefficients were determined using maltooligosaccharide standards with $D P$ ranging from 2 to 7 and were used to estimate the amounts of longer chains. Number and weight average $D P \mathrm{~s}\left(\overline{D P_{n}}\right.$ and $\overline{D P_{w}}$, respectively $)$ and polydispersity index $P$ were calculated as:

$$
\overline{D P_{n}}=\frac{\sum_{i} C_{i}}{\sum_{i}\left(\frac{C_{i}}{D P_{i}}\right)} ; \overline{D P_{w}}=\frac{\sum_{i}\left(C_{i} \times D P_{i}\right)}{\sum_{i}\left(C_{i}\right)} ; P=\frac{\overline{D P_{w}}}{\overline{D P_{n}}}
$$


Mass Spectrometry. MALDI-TOF mass spectrometry was performed on a Bruker Autoflex mass spectrometer (Bruker Spectrospin, Wissembourg, France) equipped with a nitrogen laser operating at $337 \mathrm{~nm}$. Mass spectra were recorded in reflection mode and in positive ion detection using 2,5-dihydroxybenzoic acid $(50 \mathrm{mg} / \mathrm{mL})$ as a matrix. Each sample $(1 \mu \mathrm{L})$ was mixed with $1 \mu \mathrm{L}$ of matrix and dried under diminished pressure. The matrix, 2,5-dihydroxybenzoic acid (2,5-DHB) was purchased from Sigma (France) and used without further purification.

NMR Spectroscopy. Samples were dissolved in DMSO-d6 (5 mg sample into $500 \mu \mathrm{L}$ for ${ }^{1} \mathrm{H}$ spectra and $60 \mathrm{mg}$ in $500 \mu \mathrm{L}$ for ${ }^{13} \mathrm{C}$ spectra). NMR spectra were recorded at $60^{\circ} \mathrm{C}$ in $5 \mathrm{~mm}$ NMR tubes using an AC300 spectrometer (Bruker Spectrospin) with a dual $\left({ }^{1} \mathrm{H}-{ }^{13} \mathrm{C}\right)$ probe operating at a frequency of $75.476 \mathrm{MHz}$ for ${ }^{13} \mathrm{C}$ and $300.136 \mathrm{MHz}$ for ${ }^{1} \mathrm{H} .{ }^{13} \mathrm{C}$ spectra were recorded using 90 pulses, $7500 \mathrm{~Hz}$ spectra width, 8192 data points, 0.5 s relaxation delay and up to 50000 scans. ${ }^{1} \mathrm{H}$ spectra were recorded with $2000 \mathrm{~Hz}$ spectral width, 8192 data points, $1 \mathrm{~s}$ relaxation delay and up to 16 scans. The residual signal of the solvent was used as an internal standard: $\mathrm{C} 2 \mathrm{H} 6 \mathrm{OS}$ at $2.50 \mathrm{ppm}$ and $\mathrm{C} 2 \mathrm{H} 6 \mathrm{OS}$ at $39.51 \mathrm{ppm}$. The presence of $\alpha(1,6)$ linkages was determined from ${ }^{13} \mathrm{C}$ NMR spectra according to the band assignment of Jodelet et al. ${ }^{24}$ The branching degree was calculated from the ratio between the area of the $\mathrm{H} 1 \alpha(1,4)$ band at $\delta=5.45-5.37 \mathrm{ppm}$ and that of $\mathrm{H} 1 \alpha(1,6)$ at $d=4.87-4.85 \mathrm{ppm}$ in the ${ }^{1} \mathrm{H}$ NMR spectra. ${ }^{25-26}$

Crystallization Protocol. The protocol was adapted from the procedures described by Buléon et al. ${ }^{7}$ and Imberty et al. ${ }^{13}$ Aqueous dispersions of amylose $(0.05 \% \mathrm{w} / \mathrm{v})$ were submitted to nitrogen bubbling for $20 \mathrm{~min}$ and heated in sealed vials at $150{ }^{\circ} \mathrm{C}$ for $15 \mathrm{~min}$. The solutions were then cooled down to $80^{\circ} \mathrm{C}$, filtered through $0.2 \mu \mathrm{m}$ pre-heated Sartorius Minisart RC 25 filters, heated again at $150{ }^{\circ} \mathrm{C}$ for $15 \mathrm{~min}$, cooled down to $80{ }^{\circ} \mathrm{C}$ and poured in a three-neck $100 \mathrm{~mL}$ glass balloon pre-heated at $60{ }^{\circ} \mathrm{C}$ in an oil bath. Acetone was heated at $60{ }^{\circ} \mathrm{C}$ in a separate compartment and the resulting vapors diffused through pre-heated glass tubes into the amylose solution under gentle stirring. The onset of crystallization was detected using the beam of a laser pointer which became visible as soon as crystals started to form. The mixtures were then allowed to slowly cool down to room temperature. All crystals were kept in wateracetone mother liquor and stored at $4{ }^{\circ} \mathrm{C}$.

Scanning and Transmission Electron Microscopy (SEM and TEM). Drops of dilute crystal suspensions were allowed to dry onto copper stubs. The specimens were coated with Au/Pd and observed in secondary electron mode with a JEOL JSM-6100 scanning electron microscope operating at $8 \mathrm{kV}$. Drops of dilute crystal suspensions were deposited onto glow-discharged carbon-coated copper grids and allowed to dry. The specimens were mounted on a Gatan 626 
specimen holder, quench-frozen in liquid nitrogen, transferred into the microscope and observed at low temperature, using a Philips CM200 'Cryo' microscope operating at $80 \mathrm{kV}$ for imaging and $200 \mathrm{kV}$ for diffraction. The images were recorded on Kodak SO163 films and the diffraction patterns on Fujifilm imaging plates, read with a Fujifilm BAS-1800II Bio-imaging Analyzer.

X-ray Diffraction (XRD). X-ray diffraction spectra were recorded from freshly centrifuged samples in glass capillaries using a Bruker D8 Discover diffractometer. The CuK $\alpha_{1}$ radiation $(\lambda=0.15405 \mathrm{~nm})$, produced in a sealed tube at $40 \mathrm{kV}$ and $40 \mathrm{~mA}$, was selected using a Göbel mirror parallel optics system and collimated to produce a beam with a $500 \mu \mathrm{m}$ diameter. The XRD data were collected with a two-dimensional GADDS detector during 600-s exposure times. The distance from the sample to detector was $100 \mathrm{~mm}$. All spectra were normalized at the same total integrated area between 3 and $40^{\circ}(2 \theta)$.

\section{Results}

The crystallization of short-chain amylose from dilute aqueous solutions in the presence of acetone generally yielded micrometer-size crystals. Polydisperse flower-like aggregates or more individual platelets were observed, depending on the fraction that was crystallized. In all cases, though, the single crystals exhibited an elongated platelet shape. The average lengths of the crystals prepared from each fraction are listed in Table 2.

\section{Limit dextrins from acid-hydrolyzed starch granules and short-chain maltodextrins}

LDP and LDWP fractions crystallized in the form of polydisperse flower-like aggregates with "petals" of varying length (Figures $2 a$ and $2 b$ ). The crystals were generally spindle-shaped with a round apical end. Both samples contained two families of small $(<2 \mu \mathrm{m})$ or longer (up to $5 \mu \mathrm{m}$ ) crystals. Short-chain maltodextrins (MDM) formed discoidal objects with a maximum diameter of $1 \mu \mathrm{m}$ (Figure 2c). The yield of crystallization was very low compared to that of fractions LDP and LDWP crystallized in similar conditions.

\section{Synthetic amylose}

All fractions, with the exception of SAP40-80, mainly crystallized in the form of rosettes or fan-like groups of long crystals. More individualized crystals were observed in some samples. The average length $\bar{L}$ of the crystals ranged from 1.5 to $8.5 \mu \mathrm{m}$, depending on both $\overline{D P_{n}}$ and polydispersity (Table 2). SAA fractions with $\overline{D P_{n}} \leq 17$ yielded crystals about $4 \mu \mathrm{m}$-long. With the exception of fraction SAA14-27 $(\bar{L}=4.5 \mu \mathrm{m})$, fractions with $17 \leq \overline{D P_{n}} \leq 20$ formed longer crystals $(\bar{L}=7.5 \mu \mathrm{m})$, some of them reaching $15 \mu \mathrm{m}$. SAA chains with $20 \leq \overline{D P_{n}} \leq 30$ gave 2-4 $\mu \mathrm{m}$-long crystals. Fraction SAP20-40 yielded the smallest crystals $(\bar{L}=1.5 \mu \mathrm{m})$. 
Figure 3 shows crystals prepared from six SAA fractions with $\overline{D P_{n}}$ ranging from 12 to 26 . Crystals prepared from chains with $\overline{D P_{n}} \leq 15$ were asymmetrical spindle-shaped platelets with a sharp apical end (Figure 3a). Fractions with $15 \leq \overline{D P_{n}} \leq 25$ yielded elongated platelet crystals with a flat apical end and parallelogram transverse sections (Figures $3 b, 3 c$ and $3 d$ ). They were often organized in rosettes (Figure $3 b$ ) or fan-like (Figure $3 c$ ) assemblies but a large number of individual crystals could be seen. Crystals from fraction SAA12-23 had a specific triangular shape and were often organized into rosettes (Figure $3 b$ ) and fan-like assemblies (Figure 3c). Fraction SAA14-27 yielded crystals of approximately the same size with a flat apical end but mostly organized into fan-like assemblies. Larger crystals with flat apical ends were obtained from fraction SAA17-28 (Figure 3d). Along with similar crystals prepared from fractions SAA1421 and SAA16-22 (not shown), they were among the largest and most homogeneous crystals in terms of shape and size. Fraction SAA20-29 yielded partial rosette assemblies containing crystals of varying lengths, with round apical ends (Figure 3e). Fraction SAA23-32 formed assemblies organized in two symmetrical groups of smaller and irregular spindle-shaped crystals (Figure $3 f$ ).

Crystals prepared from fractions SAP are shown in Figure 4. SAP20-40 yielded $2 \mu \mathrm{m}$-long spindle-like crystals (Figure 4a) while those formed from fraction SAP40-80 consisted of aggregates of sub-micron units with ill-defined shapes (Figure $4 b$ ).

With the exception of fractions LDWP6-42 and SAP40-80 whose crystals yielded a C-type XRD profile (Supporting Information, Figure S4), corresponding to a mixture of $A$ and $B$ crystallites, all fractions formed crystals corresponding to allomorph $A$, with main diffraction peaks at Bragg angles $2 \theta$ around $10,11,15,17,18$ and $23^{\circ}$ (Figure 5). Due to the very small amounts of material prepared for each sample, the difficulty of controlling their moisture content, and the preferred orientations in some specimens due to the anisotropic shape of the crystals, it was not possible to quantify the degree of crystallinity with precision. Therefore, it was decided to separate diffraction diagrams into three classes corresponding to degrees of crystallinity higher than $75 \%$ (symbolized by '+++'), ranging between 40 and $75 \%(++)$ and lower than $40 \%(+)$ (Table 2). Figure 5 shows the diffraction diagrams recorded from crystals prepared with fractions SAA16-22, SAA19-29 and SAP20-40, which are typical of each of the three classes. The highest crystallinity was observed for fractions with $15 \leq \overline{D P_{n}} \leq 20$ which also yielded the longest crystals, while crystals prepared from maltodextrins and SAP fractions exhibited a much lower crystallinity.

Electron diffraction diagrams were recorded from frozen-hydrated single crystals lying flat on the supporting carbon film (Figure 6a). When the long axis of the crystals was oriented parallel to the tilt axis of the holder, the specimens were tilted and diffraction patterns were recorded along different projections of the structure. Imberty et al. have previously published a series of diffraction patterns recorded from A-type crystals tilted around their long axis. ${ }^{13} \mathrm{After}$ 
indexation, the authors concluded that the patterns corresponded to projections along $h k 0$ zone axes of the monoclinic unit cell of A-amylose. Figure $6 \mathrm{~b}$ is an example of a diffraction diagram recorded from a crystal prepared with fraction SAA17-28. By comparison to the results of Imberty et al., we concluded that this pattern corresponded to the [140] zone axis and that the $c$-axis was indeed parallel to the long axis of the crystal. A thorough crystallographic analysis of the crystals from our electron diffraction data will be presented in a subsequent paper.

Complementary information on the structure of the various crystals was obtained from TEM images of negatively stained preparations. Figures $7 \mathrm{a}$ to $7 \mathrm{~d}$ show crystals prepared from limit dextrins. For fraction MDM6-22, the 400-500 nm-wide bulky particles exhibited an irregular lamellar structure (Figures $7 \mathrm{a}$ and $7 \mathrm{~b}$ ). For fraction LDP8-27, the lamellar organization was more regular and the ogival crystals grew in a direction perpendicular to the lamella plane (Figures 7c and 7d). A similar lamellar structure was observed in crystals prepared from synthetic amylose fractions such as SAA12-23 (Figures 7e and 7f). In all cases, the average thickness of one lamella was estimated to lie between 5 and $10 \mathrm{~nm}$. Although the overall morphology of the product recrystallized from fraction SAP40-80 was not clearly defined (Figure $4 b$ ), images of the negatively stained specimen showed that it consisted of a tortuous network of lamellar subunits (Figure $7 \mathrm{~g}$ ).

\section{Discussion}

\section{Crystal morphology}

Depending on the amylose fraction, the crystals were associated into rosettes or fan-like assemblies. Individual crystals likely resulted from the disruption of rosettes during sample manipulation or drying. In rosettes, crystals grew in all directions from the same nucleation point, forming spherical assemblies (Figure 3b). In the fan-like organization, crystals were symmetrically distributed around a longer central crystal. New crystals seem to have developed sequentially by heterogeneous nucleation on the largest faces of already formed crystals (Figure $3 c$ ).

The majority of crystals are elongated platelets with a flat, sharp or round apical end. When the crystals have a well-defined faceted shape, like those prepared from fractions SAA12-23 (Figure 3b), SAA14-21 or SAA17-28 (Figures S3a and S3b, respectively), the parallelogram cross-section is clearly recognizable with obtuse angles of $120 \pm 3^{\circ}$, a value that is very close to the $\gamma$ angle of the monoclinic unit cell of allomorph A $\left(123^{\circ}\right.$ for Imberty et al ${ }^{13}$ and $122^{\circ}$ for Popov et al. $\left.{ }^{21}\right)$. In addition, the cross-sections of several crystals grouped into a rosette assembly are all similar (Figure 3b, S3a and S3b, respectively). In all cases for which electron diffraction diagrams could be recorded, we concluded that the chain axis lay parallel to the long axis of the crystals and perpendicular to the lamellar structure identified in Figure 7. 
Combining these results and taking into account the monoclinic unit cell of A-amylose, we can thus propose a preliminary model that describes the morphology and structure of the A-type single crystals (Figure 8): each constituting lamella corresponds to the parallel packing of double helices in the $(a, b)$ base plane of the unit cell, the chain axis $c$ being perpendicular to the lamella but oriented opposite to the growth direction of the crystal.

This peculiar chain orientation was observed earlier by Pohu et al. in A-type amylose crystals prepared from dilute solutions of potato starch limit dextrins. ${ }^{19}$ These crystals exhibited a peculiar pine cone-like structure, with stacks of parallel lamellae symmetrically organized with respect to the long axis. Each lamella was 5-7 nm-thick, in good agreement with the length of double helices formed from DP 15 chains. In the present work, only the crystals prepared from fraction MDM seem to exhibit a similar, albeit more irregular, pine cone morphology (Figures $7 \mathrm{a}$ and $7 \mathrm{~b}$ ).

All A-type crystals prepared with less polydisperse SAA fractions and observed by TEM after negative staining appeared to be made of lamellae stacked perpendicularly to the long axis of the crystals (Figures $7 \mathrm{c}$ to $7 \mathrm{f}$ ). Considering that the pitch of an A-type amylose 6 -fold double helix is $2.10 \mathrm{~nm}$, the length of one double helix formed by chains with $15 \leq D P \leq 25$ would lie between 5.25 and $8.75 \mathrm{~nm}$ along the $c$-axis. This is in good agreement with the $5-10$ $\mathrm{nm}$ thickness of the lamellae shown in the TEM images (Figures $7 \mathrm{c}$ to $7 \mathrm{f}$ ). The longest amylose chains of our series (SAP40-80) did not form large single crystals but still showed the tendency to form lamellar subunits organized into an irregular network (Figure $7 \mathrm{~g}$ ). In this case, the correlation between chain length and crystallite thickness is difficult to establish since the subunits are ill-defined and the fraction is very polydisperse. Folding of amylose double helices is unlikely, so the chains may be part of several stacked crystallites.

Synthetic polymers ${ }^{27}$ and most polysaccharides ${ }^{28-30}$ form lamellar single crystals when recrystallized from dilute solutions and chain folding is involved for high molecular weight polymers. In this case, the chain axis is perpendicular to the base plane of the lamella, whose thickness is generally $8-10 \mathrm{~nm}$. That is also the case for all known crystalline inclusion complexes of amylose whose structure is based on the packing of single helices. ${ }^{4,31-35}$ Some crystals may be composed of a few stacked lamellae but, in most cases, the growth is lateral and not axial. In the case of A-type single crystals, even though lamellae are indeed formed by crystallization of amylose double helices, new lamellae nucleate and develop on top of each other. The lateral growth is limited while the axial growth is significantly promoted. The morphology of A-type crystals is thus rather unique for polymers but the origin of such anisotropic growth is not known yet.

\section{Influence of chain length and branching on crystal morphology}

The crystals prepared in this study were significantly larger than those used by Imberty et al. 
to model the structure of allomorph $A .{ }^{13}$ For the fractions yielding crystals with a clearly faceted shape, the crystal length ranges from 2.5 to $8.5 \mu \mathrm{m}$ and seems to depend on both $\overline{D P}$ and distribution width $(D W)$ of the fraction, the longer crystals being obtained from fractions with $17 \leq \overline{D P_{n}} \leq 22$ and $D W \leq 8$.

Figure 9 summarizes the variation in morphology of A-type crystals as a function of the $D P$ and polydispersity of amylose. We have distinguished four groups corresponding to the four main types of observed morphology: for $\overline{D P_{n}} \leq 15$, crystals are ogival platelets with sharp and thin ends; for $15<\overline{D P_{n}} \leq 22$, elongated platelets of varying length with flat apical ends; for $22 \leq \overline{D P_{n}} \leq 26$, rosettes of crystals with round apical ends; for $26 \leq \overline{D P_{n}}<40$, less well-defined spindle-like crystals, with sharp and thin ends, associated in a symmetrical fashion. We were unable to prepare single crystals from fractions with $\overline{D P_{n}} \geq 40$. Crystals prepared from LDP and LDWP fractions whose $\overline{D P_{n}}$ lies between 15 and 22 are ogival with sharp or round apical ends. They are thus different from those prepared from the SAA fractions of the same class, which could be due to the presence of branches in these limit dextrins that stem from acid hydrolysis of native starch (Table 2).

As shown in Figure 10, the polydispersity of the fractions influences the crystal size and shape. The longest crystals $(6.0 \mu \mathrm{m} \leq \bar{L} \leq 8.5 \mu \mathrm{m})$ were obtained from fractions with both $15 \leq \overline{D P_{n}} \leq 19$ and $D W \leq 8$ (SAA16-22, SAA14-21), i.e., the lowest $D W$ s of the series. Other fractions with $15 \leq \overline{D P_{n}} \leq 19$ but $D W \geq 10$, like SAA14-27, yielded shorter crystals $(4.5 \mu \mathrm{m})$. Crystals from fractions with $\overline{D P_{n}} \geq 20$ never exceed $4-5 \mu \mathrm{m}$ in length whatever the $D W$. Fractions LDP8-27 and LDP6-42, although having a $\overline{D P_{n}}$ of 16 and 21, respectively, yielded very short crystals, probably because of their high polydispersity and the presence of branches.

Branched LDP, LDWP or MDM fractions yielded crystals smaller than those obtained from SAA fractions. The effect was significant for fraction MDM with the highest branching degree $(8 \%)$. The very low crystallization yield and small size of the crystals clearly indicates that the presence of branching points limits both the nucleation and growth. Branching probably hinders the intertwining of chains into double helices and the packing of these double helices into larger crystalline arrays. In addition, branched dextrins are more soluble which may influence crystallization. In the samples prepared from fractions LDP and LDWP, two populations of crystals were observed (Figures $2 a$ and $2 b$, respectively) with length around $2 \mu \mathrm{m}$ and much below $1 \mu \mathrm{m}$, respectively. This may result from the split crystallization of the longer chains during a first stage and the shorter ones during a second stage as the amount of precipitant solubilized in the water increases. 


\section{Influence of chain length and branching on allomorphic type and crystallinity}

All SAA fractions as well as fraction SAP20-40 exhibited XRD profiles corresponding to allomorph A (Table 2). The highest crystallinity was obtained for SAA fractions with $17 \leq \overline{D P_{n}} \leq 21$. An intermediate crystallinity was found for fractions with $\overline{D P_{n}} \leq 17$ (SAA12-23) and $\overline{D P_{n}} \geq 21$ (SAA19-29, SAA23-32). The amount of amorphous phase may be higher because the nucleation domains were large, as in the case of rosettes and fan assemblies, or because a significant fraction of the chains did not crystallize. LDP8-27 is the only limit dextrin fraction belonging to this intermediate class of crystallinity. The lowest crystallinity indexes were observed for LDWP, MDM and SAP fractions characterized by a high $\overline{D P_{n}}$ (LDWP6-42, SAP20-40, SAP40-80) or a high branching degree (MDM6-22).

Crystals stemming from fractions LDWP and SAP40-80 exhibited a C-type structure which consists of a mixture of allomorphs $A$ and $B$. This may result from a split crystallization process, with a first crystallization of the longer chains $\left(\overline{D P_{n}} \geq 35\right)$ into B-crystallites in the presence of very small amount of acetone (if any) followed by the crystallization of the shorter chains in the presence of a higher amount of precipitant. Several authors have already mentioned the effect of $D P$ on the allomorphic type during crystallization in dilute solutions of short amylose chains. Longer chains would favor the formation of allomorph $B$ whereas the shorter chains would yield A-type crystals. ${ }^{7,14,18,36}$ Pfannemüller et al. ${ }^{18}$ and Gidley and Bulpin ${ }^{15}$ have shown that in aqueous solution, amylose fragments with DP 10-13 yielded A-type crystals while those with $D P>13$ led to $\mathrm{B}$ - or $\mathrm{C}$-type structures. Hizukuri et al. have also shown that, in native starch granules, the short linear segments of amylopectin organized into crystallites were longer in B-type than in A-type granules. ${ }^{14,37}$ Pohu et al. noticed a similar phenomenon during the debranching of maltodextrins at $52^{\circ} \mathrm{C},{ }^{36}$ a temperature very close to that used in this work for crystallization. The authors reported that debranching led to a split crystallization, with a first crystallization of longer chains $(D P>40)$ into B-type within the first $12 \mathrm{~h}$, followed by a second crystallization of shorter chains $(D P \cong 15)$ into A-type during the next $36 \mathrm{~h}$. In our case, the A-type crystallization was strongly favored due to the presence of the precipitating acetone.

\section{Crystal growth mechanism}

Water is known to be a good solvent of amylose at $170{ }^{\circ} \mathrm{C}$, a temperature at which the molecules lose all helical conformation. ${ }^{38}$ Creek et al. have successfully solubilized long-chain maize amylose at $10-20 \mathrm{wt} \%$ by heating at $170{ }^{\circ} \mathrm{C} .{ }^{39}$ Consequently, in our protocol, heating the fractions of short amylose chains at low concentration at $150{ }^{\circ} \mathrm{C}$ most probably resulted in a solution of non-helical individual chains. During the cooling from 150 to $60{ }^{\circ} \mathrm{C}$, amylose

chains may have stayed metastable or started entangling into double helices. The entanglement of short amylose chains in dilute aqueous solution has never been documented in the literature. The published works only report on conformational changes of high molecular 
weight amylose in concentrated solutions and gels. ${ }^{40,41}$ Here, nucleation was induced by slowly adding acetone, a precipitant of amylose, which increased supersaturation and favored the formation of nuclei by association of double helices. However, we could not assess whether the formation of double helices and crystal nuclei were separate or concomitant phenomena.

The two major types of crystal assemblies observed in this work, namely rosettes and fans, could result either from two different nucleation modes or two different types of nuclei. In rosettes, crystals simultaneously developed radially, in all directions, from a common nucleus. Although we could not clearly identify the shape of the nucleus from the SEM images, it may resemble a small sand rose and correspond to a polycrystal containing multiple twins.

In fan assemblies, the large central platelet around which crystals are distributed probably resulted from an early primary nucleation event in the homogeneous solution. Then, crystallization proceeded by heterogeneous nucleation on the two largest faces of the central crystal, yielding two neighboring crystals whose faces then became nucleation sites, and the process repeated (Figure S5).

The characteristic spindle shape of A-type amylose crystals illustrates the fact that axial growth was promoted rather than lateral growth. Assuming that the parallelogram shape of the crystal cross-section corresponds to a projection of the $(a, b)$ base plane of the monoclinic unit cell along the $c$-axis, lamellae grew by the addition of amylose double helices parallel to the other double helices and the adsorption seemed to be more favorable along the a-axis (Figure 8). As shown in the scheme of Figure 11a, in the $(a, c)$ plane of the monoclinic unit cell of A-amylose, neighboring double helices are alternately translated by $\pm c / 2$ with respect to one another along the $c$-axis, resulting in a more compact nesting. ${ }^{13}$ In the $(b, c)$ plane, the double helices are at the same level and the distance separating them is slightly larger. The addition of one double helix thus seems to be more favorable in the $(a, c)$ plane than in the $(b, c)$ plane since the resulting packing is denser. A comparison of all the crystals prepared during this work shows that their average width is around $1 \mu \mathrm{m}$. The crystal thickness is more variable but is often close to $200 \mathrm{~nm}$. Considering the parameters of the monoclinic unit cell of A-amylose $\left(a=2.083 \mathrm{~nm}, b=1.145 \mathrm{~nm}, \gamma=122^{\circ}{ }^{21}\right)$, this typical single crystal cross-section would contain about 500 and 200 unit cells along the $a$ and $b$ axes, respectively. These values may represent equilibrium dimensions of the $(a, c)$ and $(b, c)$ faces, respectively.

Since the crystals grew more extensively along the $c$-axis, we can assume that the $(a, b)$ face was less stable. The reason may be that this face has a certain roughness because i) neighboring helices are alternately shifted by $\pm c / 2$ along $c$ with respect to one another, ${ }^{13,21}$ or ii) the amylose fractions are polydisperse so that lamellae contain double helices formed by strands of different lengths and double helices with different lengths (Figure 11b). According to the two mechanisms described by Burton et al., ${ }^{42}$ the axial growth of the crystals by successive formation of lamellae in the $(a, b)$ plane can be achieved by i) the two-dimensional 
nucleation and lateral growth of steps that eventually merge into complete lamellae, or ii) the propagation of growth spirals. The latter mechanism is more frequently found as defects often exist on crystalline surfaces. ${ }^{43}$ However, it is difficult to validate one or the other mechanism in our case as the resolution of our SEM images was not sufficient to visualize defects on the apical end of the crystals organized into rosettes. In addition, the dehydration of amylose during drying, metallization and electron irradiation may have damaged the surface to some extent.

\section{Conclusion}

The results presented in this paper clearly show that molecular parameters such as chain length, polydispersity and branching degree influence both the organization of growing A-type amylose crystals (rosettes or fans) and the morphology of individual crystals. The longest (around $10 \mu \mathrm{m}$ ) and most clearly faceted elongated platelets were prepared with narrow fractions of linear synthetic amylose. Typically, within the panel of fractions available in this study, the optimum chain length lay between 17 and 20 with a distribution width of around 6-7. The resulting single crystals are among the largest ones ever synthesized from a polymer in dilute solution. In addition, their supramolecular organization is very peculiar and does not resemble that usually observed in lamellar polymer crystals.

The longest amylose chains available, with $\overline{D P}>40$ and a high polydispersity, did not yield single crystals but ill-defined networks of smaller crystallites. The preparation of narrow fractions with a $\overline{D P}$ ranging between 30 and 70 may help determine more precisely the limit above which it is not possible to form A-type single crystals.

A subsequent part of this study will consist in evaluating the role of operating parameters such as amylose concentration, crystallization temperature and nature of the precipitant. Work is also in progress to analyze electron diffraction datasets from the most clearly faceted A-type single crystals and unambiguously characterize the crystal habit. While we have presented her preliminary results on the crystal growth, further investigations are required to confirm if a mechanism based on step propagation or spiral growth applies.

Acknowledgment. This research project has been partly supported by a Marie Curie Early Stage Research Training Fellowship of the European Community's $6^{\text {th }}$ Framework Programme, under contract $\mathrm{n}^{\circ} \mathrm{MEST}-\mathrm{CT}-2004-503322$. We thank D. Dupeyre and C. Lancelon-Pin (CERMAV) for assistance with scanning electron microscopy as well as A. Heyraud (CERMAV) for the gift of samples of fractionated maltodextrins. The technical support of B. Pontoire (INRA) for X-ray diffraction, I. Jeacomine (CERMAV) for NMR spectroscopy and $S$. Boullanger (CERMAV) for mass spectrometry is also acknowledged. 


\section{References}

(1) Buléon, A.; Colonna, P.; Planchot, V.; Ball, S. Int. J. Biol. Macromol. 1998, 23, 85-112.

(2) Paris, M.; Bizot, H.; Emery J.; Buzaré, J-Y.; Buléon, A. Carbohydr. Polym. 1999, 39, 327339.

(3) Zobel, H.F. Starch/Stärke 1988, 40, 1-7.

(4) Buléon, A.; G. Véronèse, G.; Putaux, J-L. Aust. J. Chem. 2007, 60, 706-718.

(5) Leloup, V. M.; Colonna, P.; Ring, S. G.; Roberts, K.; Wells, B. Carbohydr. Polym. 1992, 18, 189-197.

(6) Gidley, M. J. Macromolecules 1989, 22, 351-358.

(7) Buléon, A.; Duprat, F.; Booy, F. P.; Chanzy, H. Carbohydr. Polym. 1984, 4, 161-173.

(8) Brisson, J.; Chanzy, H.; Winter, W. Int. J. Biol. Macromol. 1991, 13, 31-39.

(9) Kainuma, K.; French, D. Biopolymers 1972, 11, 2241-2250.

(10) Wu, H. C. H.; Sarko, A. Carbohydr. Res. 1978a, 61, 7-25.

(11) Wu, H. C. H.; Sarko, A. Carbohydr. Res. 1978b, 61, 27-40.

(12) Imberty, A.; Perez, S. Biopolymers 1988, 27, 1205-1221.

(13) Imberty, A.; Chanzy, H.; Perez, S.; Buléon, A.; Tran, V. J. Mol. Biol. 1988, 201, 365-384.

(14) Hizukuri, S.; Kaneko, T.; Takeda, Y. Biochim. Biophys. Acta 1983, 760, 188-191.

(15) Gidley, M. J.; Bulpin, P. V. Carbohydr. Res. 1987, 161, 291-300.

(16) Kitamura, S.; Yunokawa, H.; Mitsuie, S.; Kuge, T. Polym. J. 1982, 14(2), 93-99.

(17) Potocki de Montalk, G.; Remaud-Siméon, M.; Willemot, R.-M.; Planchot, V.; Monsan, P. J. Bacteriol. 1999, 181, 375-381.

(18) Pfannemüller, B. Int. J. Biol. Macromol. 1987, 9, 105-108.

(19) Pohu, A.; Putaux, J-L., Planchot, V.; Colonna, P.; Buléon, A. Biomacromolecules 2004, $5,119-125$.

(20) Popov, D.; Burghammer, M.; Buléon, A.; Montesanti, N.; Putaux, J-L.; Riekel, C. Macromolecules 2006, 39, 3704-3706.

(21) Popov, D.; Buléon, A.; Burghammer, M.; Chanzy, H.; Montesanti, N.; Putaux, J-L.; Potocki-Véronèse, G.; Riekel, C., Macromolecules 2009, 42, 1167-1174.

(22) Robin, J-P.; Mercier, C.; Duprat, F.; Charbonnière, R.; Guilbot, A. Cereal Chem. 1974, 51, 389-406.

(23) Koch, K.; Andersson, R.; Aman, P. J. Chromatogr. 1998, 800, 199-206.

(24) Jodelet, A.; Rigby, N. M.; Colquhoun, I. J. Carbohydr. Res. 1998, 312, 139-151.

(25) Gidley, M. J. Carbohydr. Res. 1985, 139, 85-93.

(26) Nilsson G.S.; Bergquist, K.E.; Nilsson, U.; Gorton, L. Starch/Stärke 1996, 48, 352-357.

(27) Wunderlich, B. in "Macromolecular Physics", Academic Press, New York, 1973, vol. 1, pp 232-258.

(28) Buléon, A.; Chanzy, H. J. Polym. Sci. Part B Polym. Phys. 1978, 16, 833-839.

(29) Guizard, C.; Chanzy, H.; Sarko, A. Macromolecules 1984, 17, 100-107. 
(30) Persson, E.; Domard, A.; Chanzy, H. Int. J. Biol. Macromol. 1992, 14, 221-224.

(31) Buléon, A.; Delage, M.M.; Brisson, J.; Chanzy, H. Int. J. Biol. Macromol. 1990, 12, 25-33.

(32) Brisson, J.; Chanzy, H.; Winter, W. T. Int. J. Biol. Macromol. 1991, 13, 31-39.

(33) Helbert, W.; Chanzy, H. Int. J. Biol. Macromol. 1994, 16, 207-213.

(34) Hullemann, S. H.; Helbert, W.; Chanzy, H. Int. J. Biol. Macromol. 1996, 18, 115-122.

(35) Cardoso, M. B.; Putaux, J-L.; Nishiyama, Y.; Helbert, W.; Hÿtch, M.; Silveira, N. P.; Chanzy, H. Biomacromolecules 2007, 8, 1319-1326.

(36) Pohu, A.; Putaux, J-L., Planchot, V.; Colonna, P.; Buléon, A. Biomacromolecules 2004, 5, 1792-1798.

(37) Hizukuri, S.; Kaneko, T.; Takeda, Y. Carbohydr. Res. 1983, 147, 342-347.

(38) Moates, G. K.; Noel, T. R.; Parker, R.; Ring, S. G. Carbohydr. Res. 1997, 298, 327-333.

(39) Creek, J. A.; Ziegler, G. R.; Runt, J. Biomacromolecules 2006, 7, 761-770.

(40) Benczedi, D.; Tomka, I.; Escher, F. Macromolecules 1998, 31, 3055-3061.

(41) Miles, M. J.; Morris, V. J.; Ring, S. Carbohydr. Res. 1985, 135, 257-269.

(42) Burton, W. K.; Cabrera, N.; Frank, C. F. Phil. Trans. Roy. Soc. Lond. A, Math. Phys. Sci. 1951, 243, 299-358.

(43) Boistelle, R; Astier, J-P. J. Cryst. Growth 1988, 90, 14-30. 


\section{TABLES AND FIGURES}

Table 1. Nomenclature and molecular characteristics of the fractions used in this study: limit dextrins from wrinkled pea starch (LDWP), limit dextrins from potato starch (LDP), maize maltodextrins from maize (MDM), synthetic amylose prepared with amylosucrase (SAA) and starch phosphorylase (SAP).

\begin{tabular}{|c|c|c|c|c|c|c|c|}
\hline source & $D P_{\min }$ & $D P_{\max }$ & $D W^{\mathrm{d}}$ & $\overline{D P_{n}}$ & $\overline{D P_{w}}$ & $P=\frac{\overline{D P_{w}}}{\overline{D P_{n}}}$ & \% branching \\
\hline LDWPa $^{a}$ & 6 & 42 & 36 & 21.2 & 25.2 & 1.187 & 4 \\
\hline $\mathrm{LDP}^{\mathrm{b}}$ & 8 & 27 & 19 & $16^{c}$ & n. d. & n. d. & 5 \\
\hline $\mathrm{MDM}^{\mathrm{b}}$ & 6 & 22 & 16 & $11^{\mathrm{c}}$ & n. d. & n. d. & 8 \\
\hline $\mathrm{SAA}^{\mathrm{a}}$ & 9 & 15 & 6 & 12.0 & 12.1 & 1.010 & n. a. \\
\hline$S A A^{a}$ & 12 & 23 & 11 & 16.4 & 16.7 & 1.013 & n. a. \\
\hline $\mathrm{SAA}^{\mathrm{a}}$ & 14 & 21 & 7 & 17.5 & 17.6 & 1.005 & n. a. \\
\hline $\mathrm{SAA}^{\mathrm{a}}$ & 14 & 27 & 13 & 18.7 & 19.0 & 1.016 & n. a. \\
\hline$S A A^{a}$ & 16 & 22 & 6 & 19.2 & 19.2 & 1.004 & n. a. \\
\hline $\mathrm{SAA}^{\mathrm{a}}$ & 17 & 28 & 11 & 20.9 & 21.0 & 1.006 & n. a. \\
\hline $\mathrm{SAA}^{\mathrm{a}}$ & 19 & 29 & 10 & 22.3 & 22.4 & 1.005 & n. a. \\
\hline$S A A^{a}$ & 23 & 32 & 9 & 26.0 & 26.1 & 1.003 & n. a. \\
\hline$S A P^{b}$ & 20 & 40 & 20 & $32^{(\mathrm{c})}$ & n. d. & n. d. & n. a. \\
\hline SAP & 40 & 80 & 40 & $55^{(\mathrm{c})}$ & n. d. & n. d. & n. a. \\
\hline
\end{tabular}

a HPAEC-PAD; ${ }^{\mathrm{b}}$ MALDI TOF-MS; ${ }^{\mathrm{c}} D P$ of the peak with maximum height;

${ }^{d}$ distribution width $D W=D P_{\max }-D P_{\min } ; \mathrm{n}$. d.: not determined; n. a.: not applicable. 
Table 2. Average crystal length $\bar{L}$ and standard deviation std, allomorphic type and crystallinity index of amylose crystals prepared from different fractions. The diffraction diagrams were separated into three classes corresponding to degrees of crystallinity higher than $75 \%(+++)$, ranging between 40 and $75 \%(++)$ and lower than $40 \%(+)$.

\begin{tabular}{|c|c|c|c|c|}
\hline fraction & $\overline{D P_{n}}$ & $\bar{L}(\mu \mathrm{m})[s t d(\mu \mathrm{m})]$ & allomorph & $\begin{array}{l}\text { crystallinity } \\
\text { index }\end{array}$ \\
\hline LDWP6-42 & 21.2 & $0.8 / 2.2[0.7 / 1.3]$ & C & + \\
\hline LDP8-27 & $16^{a}$ & $0.7 / 3.2[0.5 / 0.9]$ & $A$ & ++ \\
\hline MDM6-22 & $11^{\mathrm{a}}$ & $0.7[0.2]$ & $A$ & + \\
\hline SAA9-15 & 12.0 & $4.2[1.4]$ & $A$ & n. d. \\
\hline SAA12-23 & 16.4 & $4.2[1.0]$ & $A$ & ++ \\
\hline SAA14-21 & 17.5 & $7.0[2.1]$ & $A$ & +++ \\
\hline SAA14-27 & 18.7 & $4.5[0.7]$ & A & +++ \\
\hline SAA16-22 & 19.2 & $8.5[2.4]$ & A & +++ \\
\hline SAA17-28 & 20.9 & $4.8[0.6]$ & $A$ & +++ \\
\hline SAA19-29 & 22.3 & $3.7[0.9]$ & $A$ & ++ \\
\hline SAA23-32 & 26.0 & $2.5[0.7]$ & $A$ & ++ \\
\hline SAP20-40 & $32^{a}$ & $1.6[0.3]$ & $A$ & + \\
\hline SAP40-80 & $55^{\mathrm{a}}$ & n. a. & $\mathrm{C}$ & + \\
\hline
\end{tabular}

${ }^{\text {a }} D P$ of the peak with maximum height; $\mathrm{n}$. a.: not applicable 

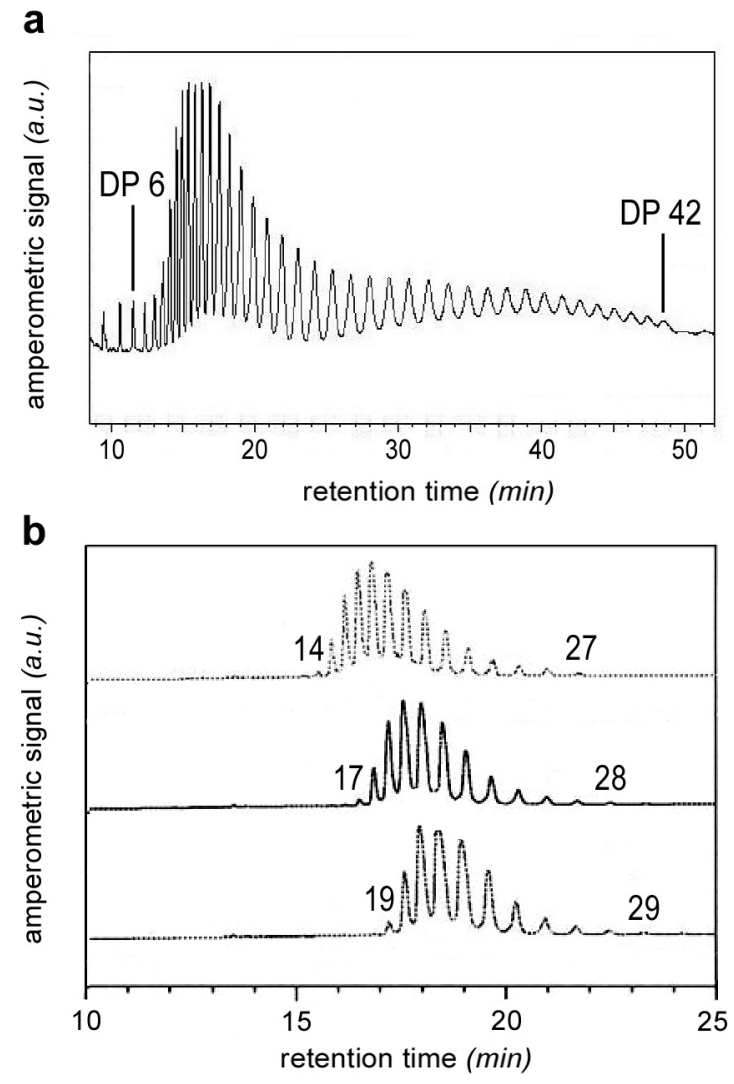

Figure 1. HPAEC-PAD chromatograms of fraction LDWP6-42 (a) and some representative SAA fractions (b). The minimum and maximum degrees of polymerization of the $\alpha(1,4)$-linked glucosyl unit chains present in the fractions are given above the peaks. 

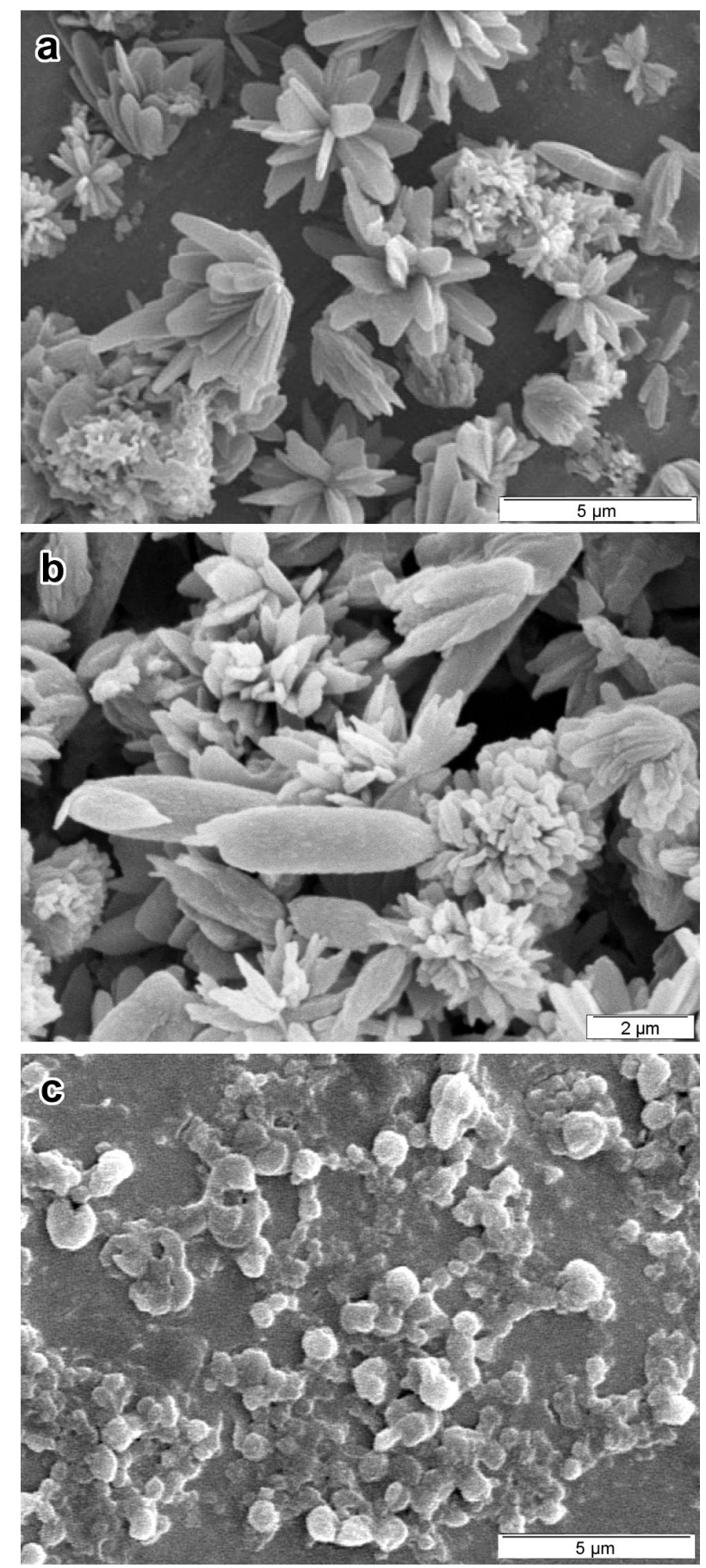

Figure 2. SEM images of crystals prepared using limit dextrins from fractions LDP8-27 (a), LDWP6-42 (b) and MDM6-22 (c). 

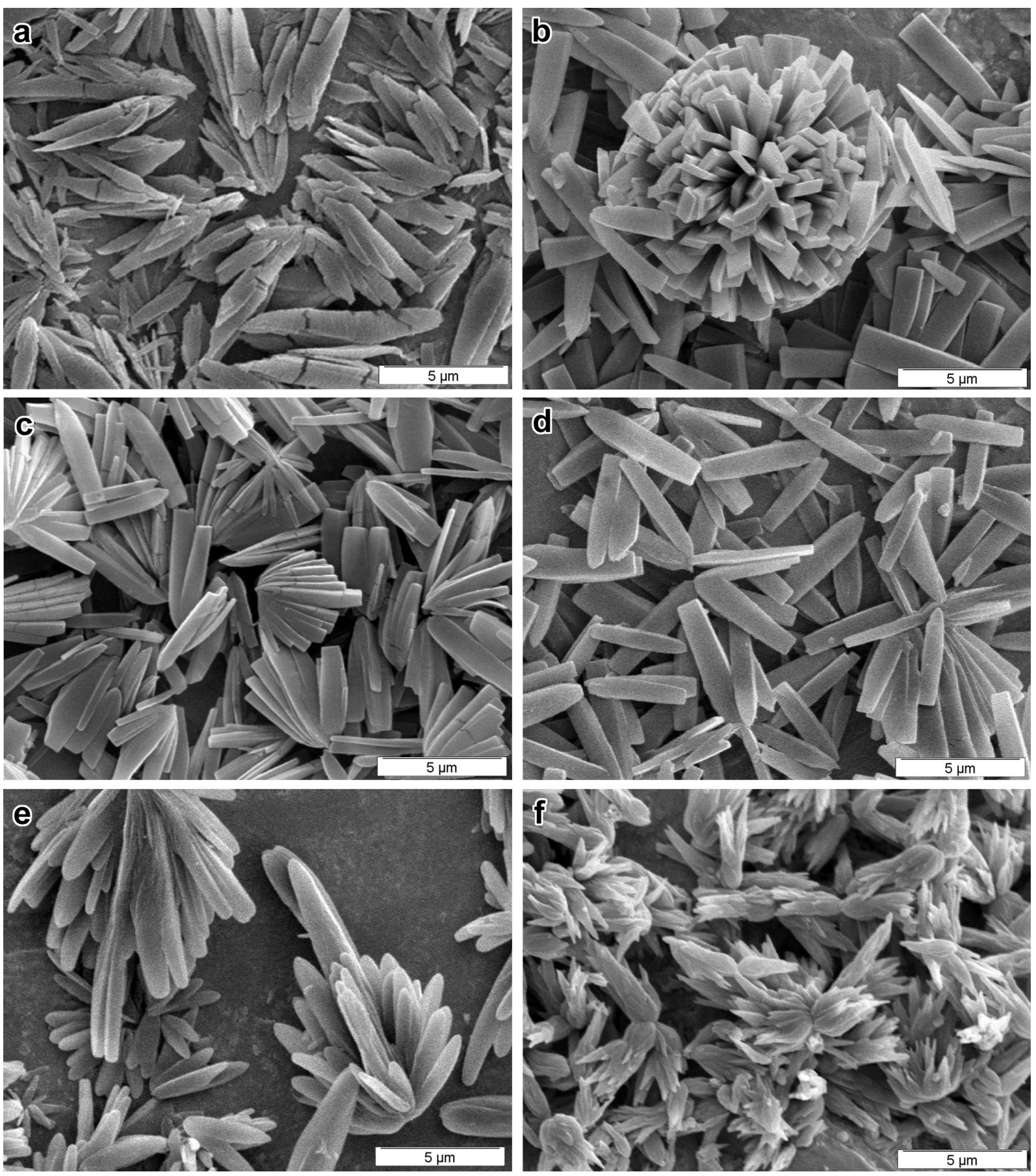

Figure 3. SEM images of crystals prepared from amylose synthesized in vitro by amylosucrase:

a) SAA9-15; b) SAA12-23; c) SAA14-27; d) SAA17-28; e) SAA20-29; f) SAA23-32. 

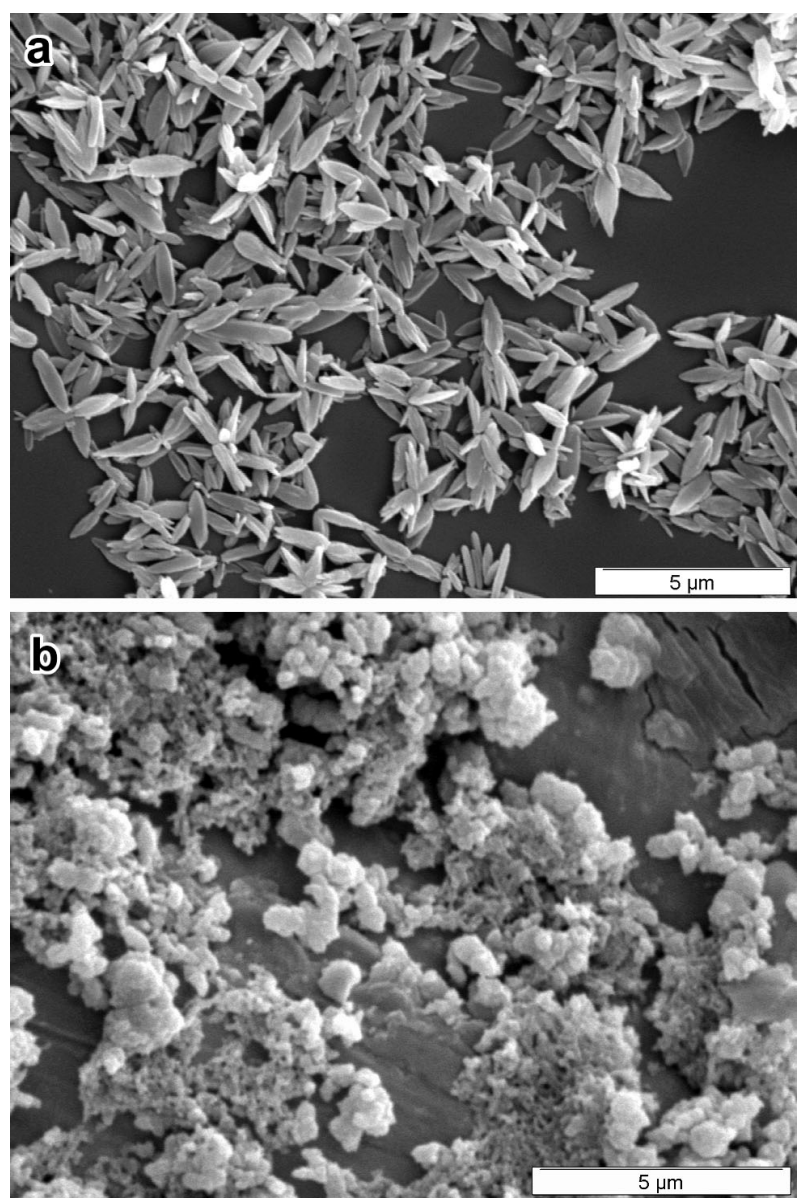

Figure 4. SEM images of crystals prepared from fractions of linear amylose synthesized in vitro with starch phosphorylase: a) SAP20-40; b) SAP40-80. 


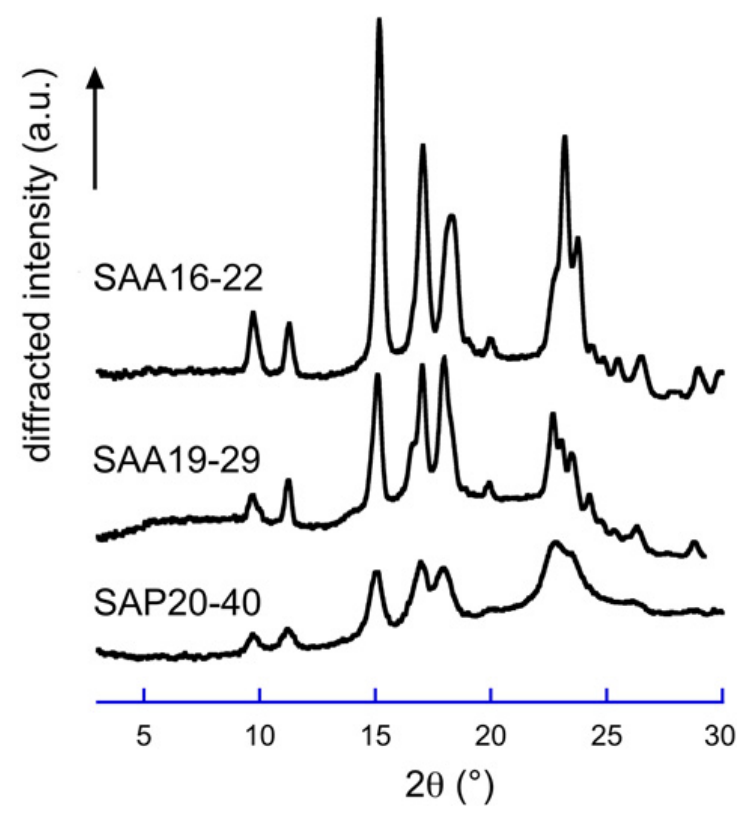

Figure 5. X-ray powder diffraction diagrams of crystals prepared from fractions SAA16-22, SAA19-29 and SAP20-40.
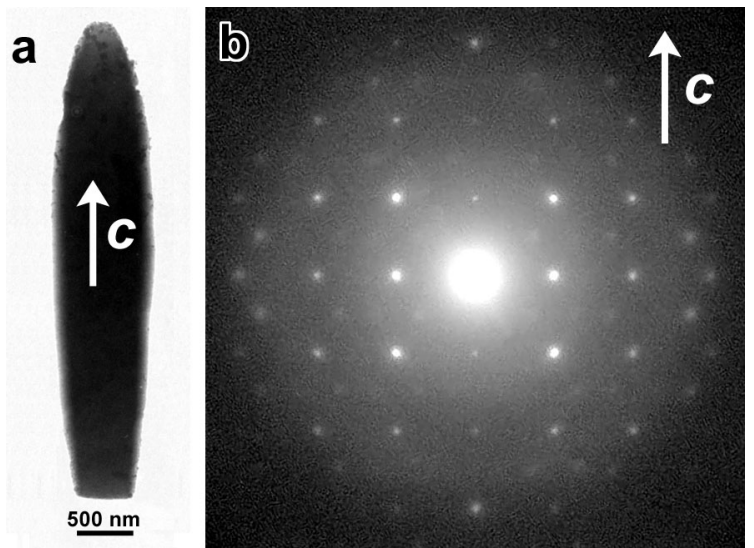

Figure 6. a) TEM image of a single crystal of A-amylose prepared from fraction SAA17-28; b) example of electron diffraction pattern properly oriented with respect to the crystal and corresponding to the [140] zone axis of the monoclinic unit cell. ${ }^{13}$ 


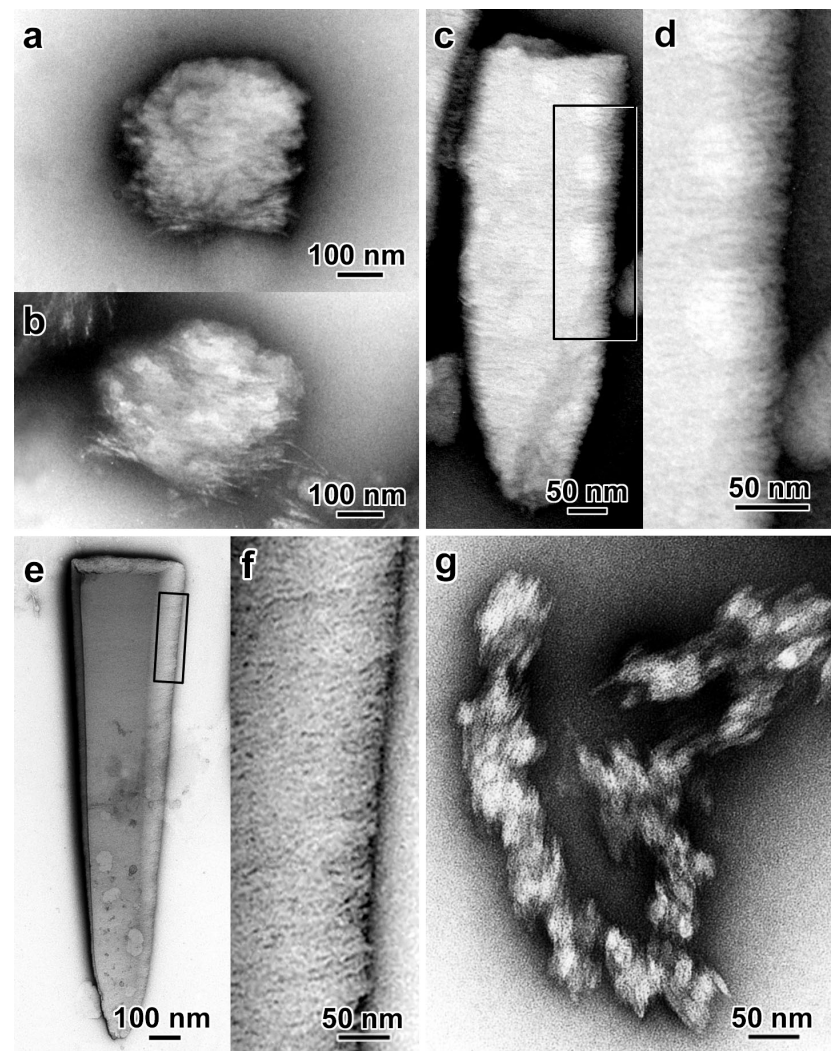

Figure 7. TEM images of negatively stained A-type single crystals prepared from fractions MDM6-22 (a,b), LDP8-27 (c,d), SAA12-23 (e,f) and SAP40-80 (g). d and f are enlargements of the regions delimited by black rectangles in $c$ and e, respectively.

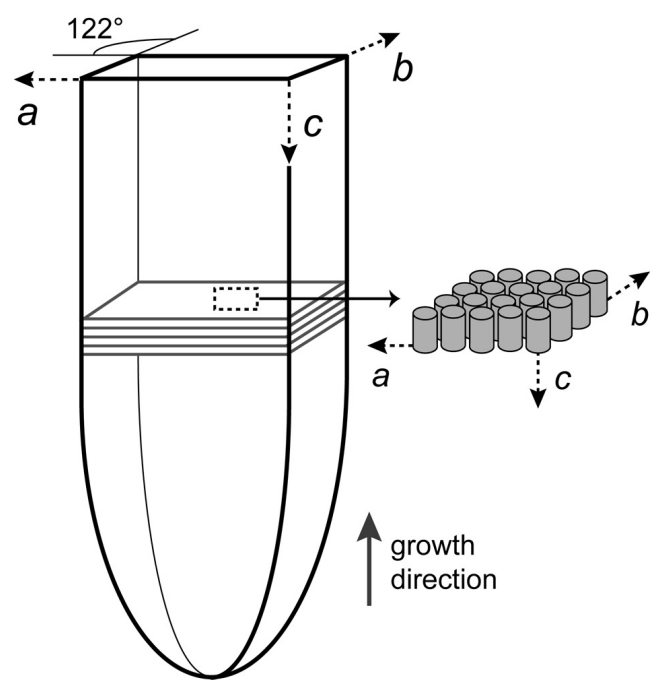

Figure 8. Scheme describing the orientation of the double helices in the long A-amylose single crystals. The grey cylinders correspond to amylose double helices. 

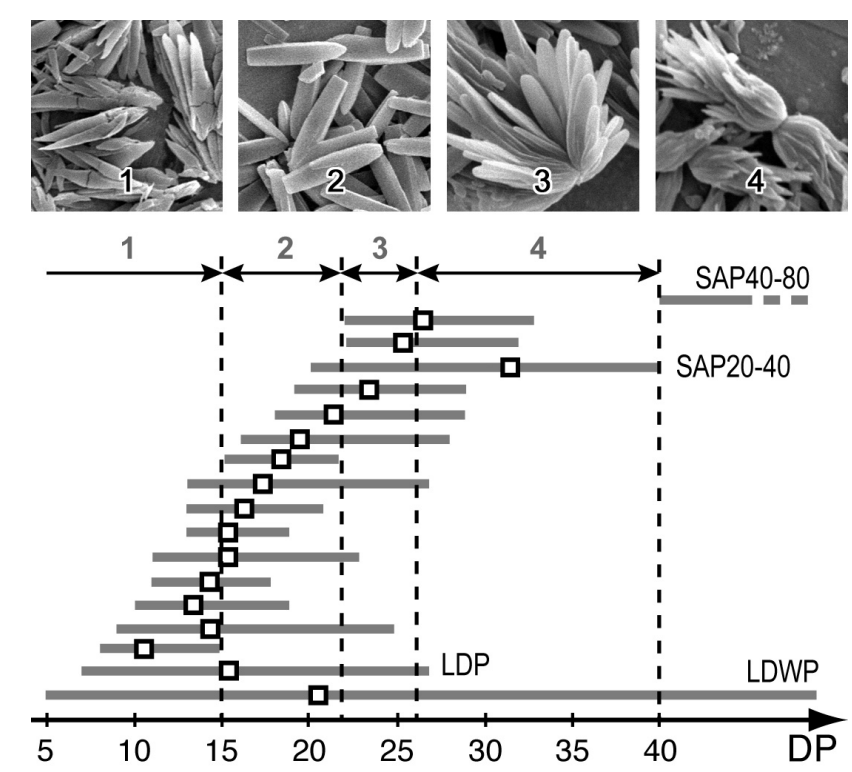

Figure 9. Variation in the morphology of A-type crystals as a function of the amylose $D P$. Each fraction is characterized by $\overline{D P_{n}}$ (indicated by an empty square) and by a horizontal bar indicating the distribution width $D W$. Except where indicated (SAP, LDP and LDWP), all fractions are from the SAA series. Four groups were distinguished, delimited by dashed lines: (1) $\overline{D P_{n}} \leq 15$; (2) $15 \leq \overline{D P_{n}} \leq 22$; (3) $22 \leq \overline{D P_{n}} \leq 26$; (4) $\overline{D P_{n}} \geq 26$. The SEM images above show crystals with shapes that are typical from the corresponding group.

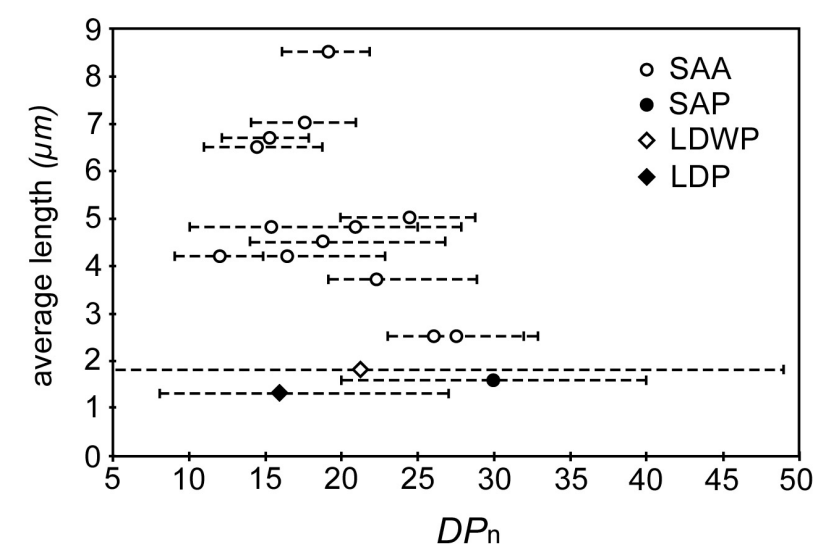

Figure 10. Average length of the crystals as a function of $\overline{D P_{n}}$. The horizontal dashed bars indicate the distribution width $D W$ of each fraction. 

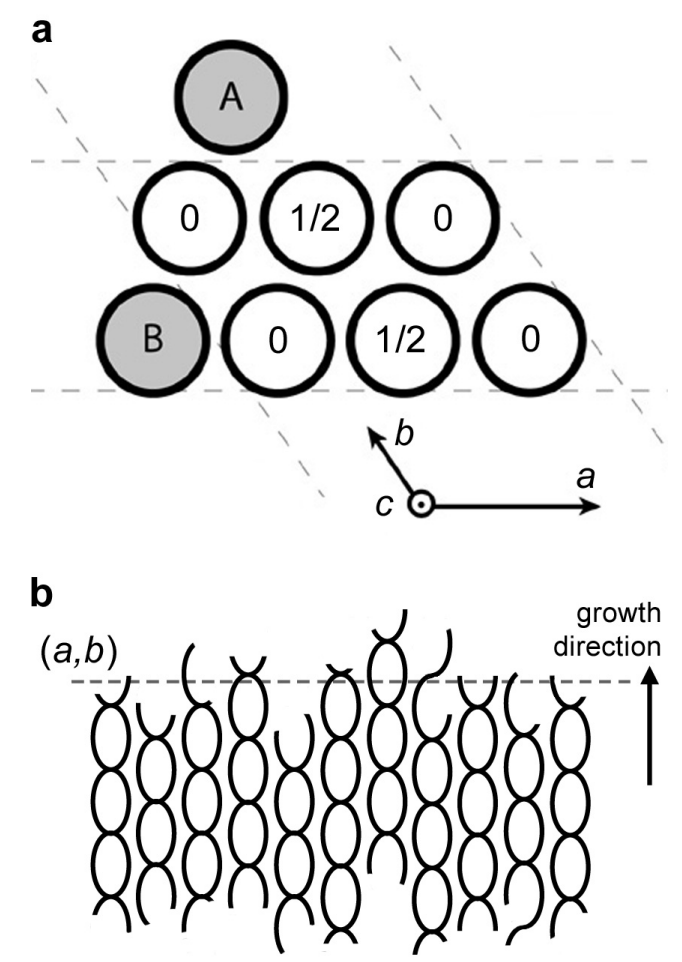

Figure 11. a) Projection of the A-amylose unit cell along the $c$-axis. ${ }^{12}$ The circles correspond to the cross-sections of double helices at 0 or $1 / 2$ level along $c$. Grey A and B disks represent possible positions of double helices that adsorb on the growing faces. b) Longitudinal view of a crystalline lamella showing the parallel packing of double helices and the possible roughness of the $(a, b)$ face due to variations of chain $D P$ and irregularities in the packing of double helices. 
SUPPORTING INFORMATION

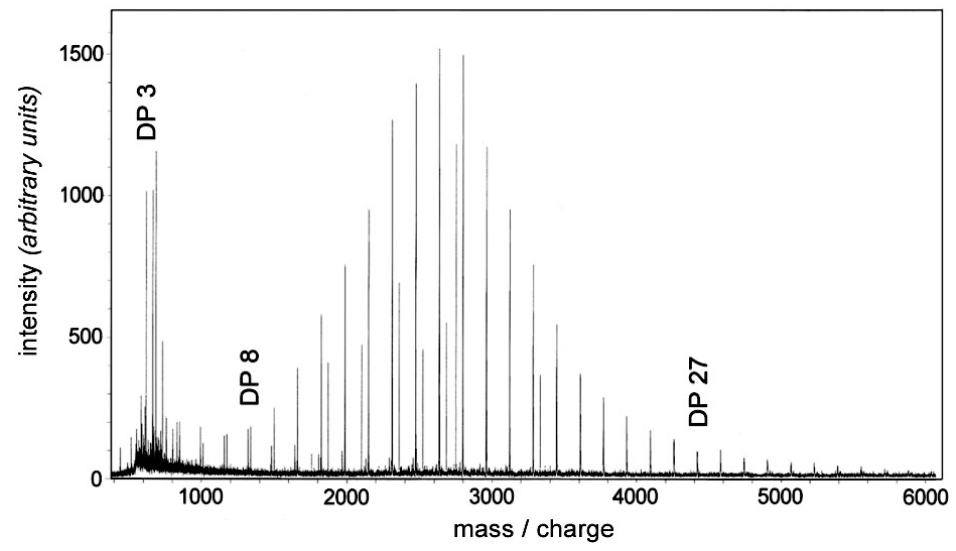

Figure S1. HPAEC-PAD chromatogram of potato starch limit dextrins (fraction LDP8-27).

a

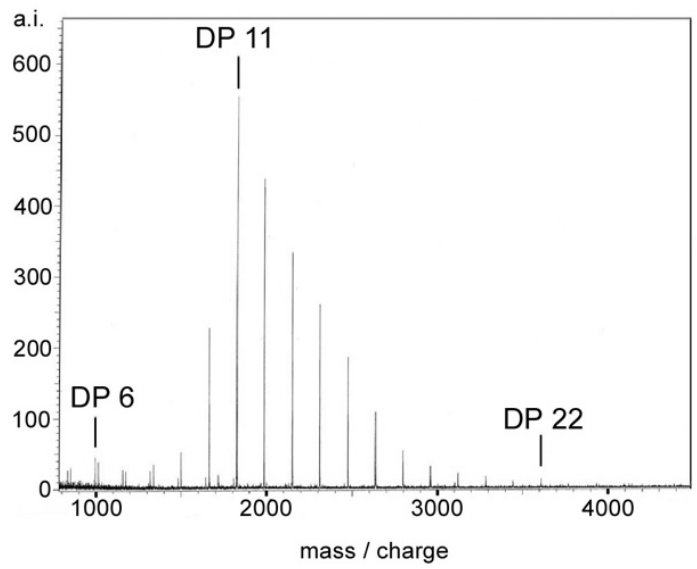

b

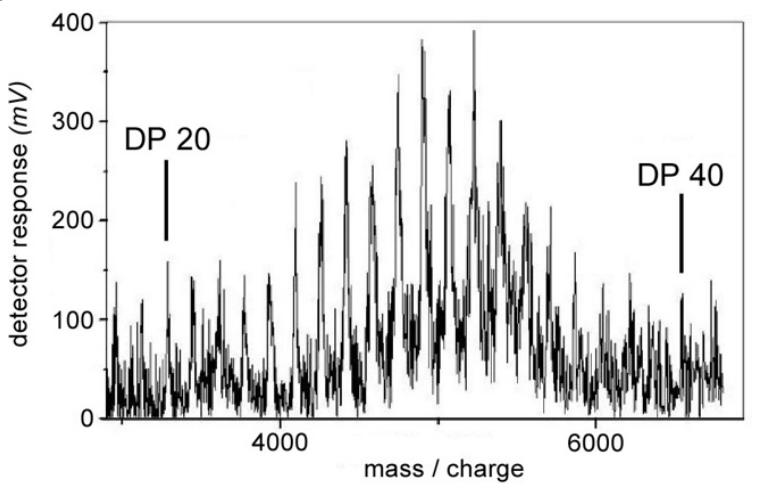

Figure S2. MALDI-TOF spectra of maize maltodextrins (a - fraction MDM6-22) and amylose synthesized by phosphorylase (b - fraction SAP20-40). 

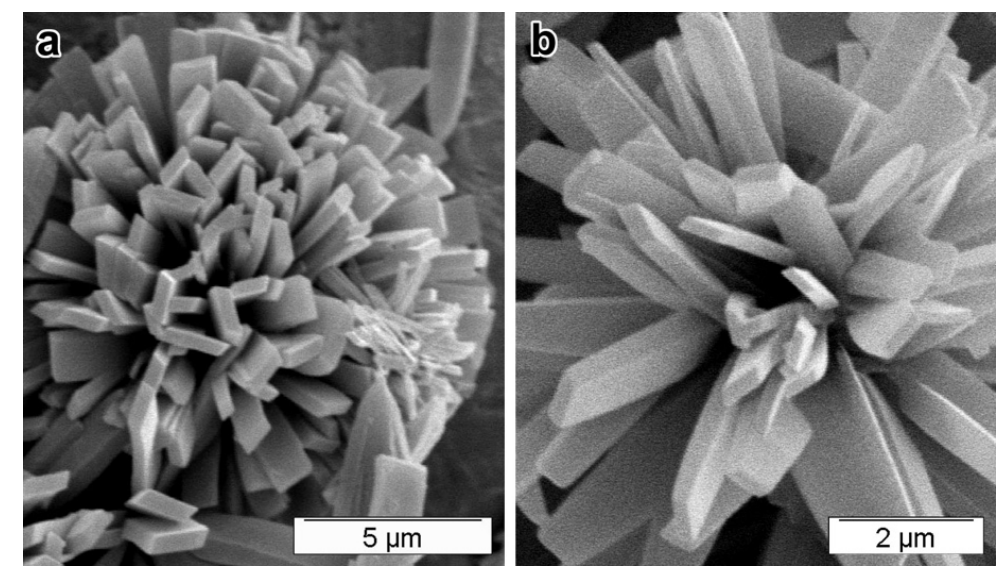

Figure S3. SEM images of rosette-like assemblies of A-type crystals prepared from fractions SAA14-21 (a) and SAA17-28 (b). The parallelogram cross-sections are similar in both cases.

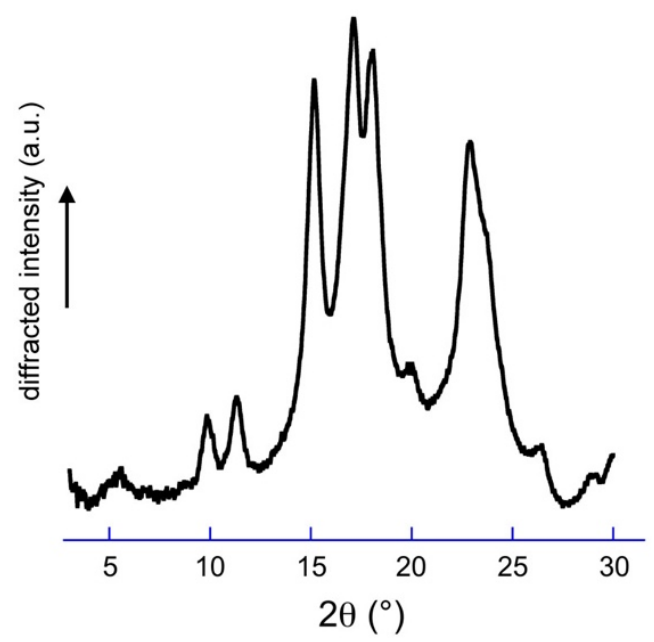

Figure S4. C-type X-ray diffraction diagram of crystals prepared from fraction LDWP6-42.

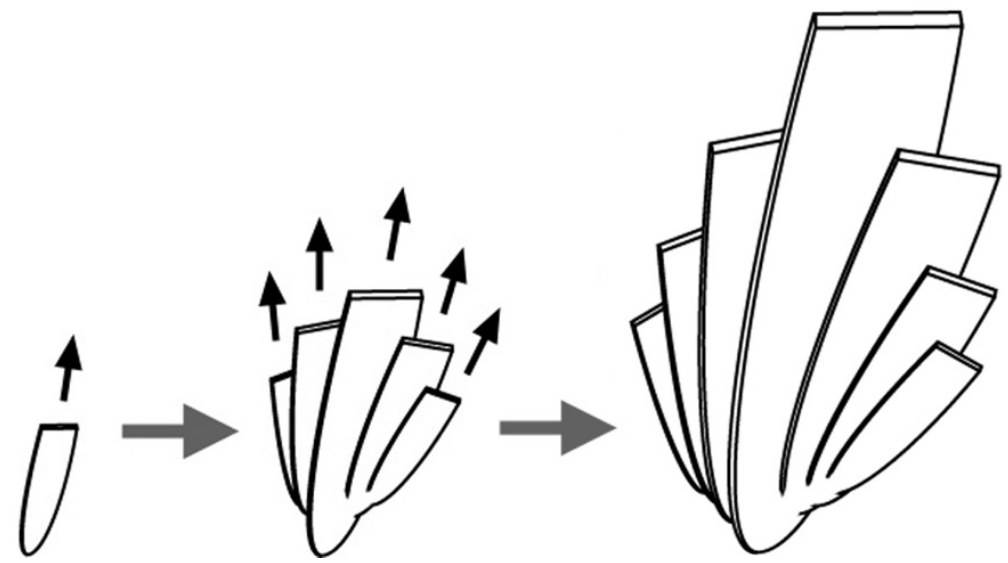

Figure S5. Scheme describing the growth of fan assemblies of crystals. 